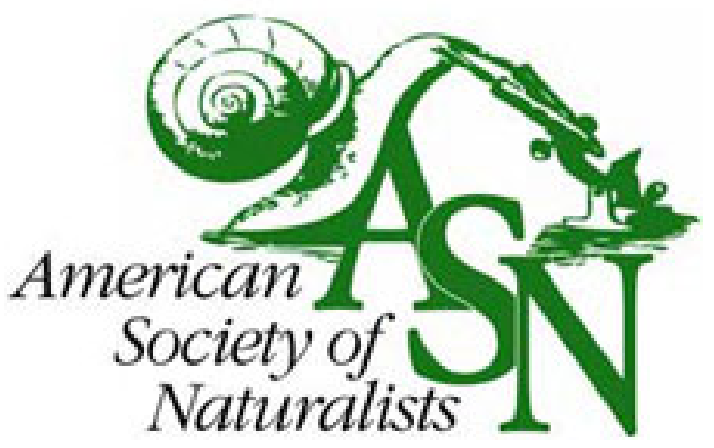

The University of Chicago

Dispersal Dynamics in Food Webs.

Author(s): Carlos J. Melián, Vlastimil Křivan, Florian Altermatt, Petr Starý, Loïc Pellissier, and Frederik De Laender

Source: The American Naturalist, Vol. 185, No. 2 (February 2015), pp. 157-168

Published by: The University of Chicago Press for The American Society of Naturalists

Stable URL: http://www.jstor.org/stable/10.1086/679505

Accessed: 22/07/2015 04:31

Your use of the JSTOR archive indicates your acceptance of the Terms \& Conditions of Use, available at http://www.jstor.org/page/info/about/policies/terms.jsp

JSTOR is a not-for-profit service that helps scholars, researchers, and students discover, use, and build upon a wide range of content in a trusted digital archive. We use information technology and tools to increase productivity and facilitate new forms of scholarship. For more information about JSTOR, please contact support@jstor.org.

The University of Chicago Press, The American Society of Naturalists, The University of Chicago are collaborating with JSTOR to digitize, preserve and extend access to The American Naturalist. 


\title{
Dispersal Dynamics in Food Webs
}

\author{
Carlos J. Melián, ${ }^{1, \star}, \dagger$ Vlastimil Křivan, ${ }^{2,3, *}$ Florian Altermatt, ${ }^{4}$ Petr Starý, ${ }^{2}$ Loïc Pellissier, ${ }^{5}$ and \\ Frederik De Laender
}

1. Fish Ecology and Evolution Department, Center for Ecology, Evolution and Biogeochemistry, Swiss Federal Institute of Aquatic Science and Technology, Kastanienbaum, Switzerland; and National Center for Ecological Analysis and Synthesis University of California, Santa Barbara, California; 2. Institute of Entomology, Biology Centre of the Academy of Sciences of the Czech Republic, České Budějovice, Czech Republic; 3. Faculty of Science, University of South Bohemia, České Budějovice, Czech Republic; 4. Department of Aquatic Ecology, Swiss Federal Institute of Aquatic Science and Technology, Düberndorf, Switzerland; and Institute of Evolutionary Biology and Environmental Studies, University of Zürich, Zurich, Switzerland; 5. Arctic Research Centre, Department of Bioscience, Aarhus University, Aarhus, Denmark; 6. Research Unit in Environmental and Evolutionary Biology, Université de Namur, Namur, Belgium Submitted October 23, 2013; Accepted October 2, 2014; Electronically published January 14, 2015

Online enhancement: appendixes. Dryad data: http://dx.doi.org/10.5061/dryad.06bb5.

AвsтRACт: Studies of food webs suggest that limited nonrandom dispersal can play an important role in structuring food webs. It is not clear, however, whether density-dependent dispersal fits empirical patterns of food webs better than density-independent dispersal. Here, we study a spatially distributed food web, using a series of population-dispersal models that contrast density-independent and density-dependent dispersal in landscapes where sampled sites are either homogeneously or heterogeneously distributed. These models are fitted to empirical data, allowing us to infer mechanisms that are consistent with the data. Our results show that models with densitydependent dispersal fit the $\alpha, \beta$, and $\gamma$ tritrophic richness observed in empirical data best. Our results also show that density-dependent dispersal leads to a critical distance threshold beyond which site similarity (i.e., $\beta$ tritrophic richness) starts to decrease much faster. Such a threshold can also be detected in the empirical data. In contrast, models with density-independent dispersal do not predict such a threshold. Moreover, preferential dispersal from more centrally located sites to peripheral sites does not provide a better fit to empirical data when compared with symmetric dispersal between sites. Our results suggest that nonrandom dispersal in heterogeneous landscapes is an important driver that shapes local and regional richness (i.e., $\alpha$ and $\gamma$ tritrophic richness, respectively) as well as the distancedecay relationship (i.e., $\beta$ tritrophic richness) in food webs.

Keywords: $\alpha, \beta$, and $\gamma$ tritrophic richness, distance-decay relationship, metacommunity dynamics, spatial food webs, heterogeneous landscape.

\section{Introduction}

Species coexistence in metacommunities (i.e., groups of spatially structured communities connected by dispersal;

* Shared first authorship.

† Corresponding author; e-mail: carlos.melian@eawag.ch.

Am. Nat. 2015. Vol. 185, pp. 157-168. (C) 2015 by The University of Chicago. 0003-0147/2015/18502-55052\$15.00. All rights reserved.

DOI: $10.1086 / 679505$
Leibold et al. 2004) depends on both trophic interactions within each local community and patterns of dispersal between these communities (Araújo and Luoto 2007; Boulangeat et al. 2012). Metapopulation theory (Levins 1962; Hanski 1999) predicts that despite local extinctions, a population can survive in a fragmented landscape consisting of several patches. In metacommunities with two trophic levels, intermediate dispersal rates can stabilize otherwise unstable resource-consumer population dynamics (Huffaker 1958). Theoretical research (e.g., Murdoch et al. 2003; Briggs and Hoopes 2004; Krivan 2008) showed that the necessary conditions for such global species coexistence in resource-consumer metacommunities are either differences in local population dynamics or differences in dispersal dynamics between patches. Moreover, dispersal rates cannot be either too low (because low dispersal rates do not rescue populations from global extinction) or too high to avoid synchronization of population dynamics (Gouhier et al. 2010). However, for metacommunities with a resource-consumer-predator food chain in each patch, Koelle and Vandermeer (2005) showed that an increase in dispersal rates can reduce synchrony in population dynamics. In multitrophic communities, a peak in food web complexity and species diversity arises for intermediate dispersal rates (Pillai et al. 2011).

Most of the theoretical work on metacommunities assumes density-independent (e.g., random) dispersal between patches. Theoretical work on simple di- and tritrophic metacommunities shows that density-dependent dispersal in the direction of higher fitness promotes species coexistence by weakening competition (Holt and Hoopes 2005; Krrivan 2014). Yet it is not clear whether densityindependent or density-dependent dispersal better predicts the empirical patterns of food webs (Koelle and Vandermeer 2005; Amarasekare 2008; Rezende et al. 2009; Massol 
et al. 2011; Thuiller et al. 2013). This knowledge gap is particularly relevant when one confronts dispersal models with extensive data sets on geographically distant food webs (Massol et al. 2011; Smith et al. 2011; Kissling et al. 2012).

Metacommunities are often characterized by three indexes: $\alpha$ richness measures local richness (i.e., the number of species at a site), $\gamma$ richness measures regional richness (i.e., the number of species at the region/landscape), and $\beta$ richness measures changes in community composition by comparing species composition at two or more sites. A measure that is particularly relevant for describing metacommunities is the distance-decay relationship in community similarity (Nekola and Peter 1999; Morlon et al. 2008), expressed as the dependence of the $\beta$ richness on geographic distance between sites. It is known that the distance-decay relationship is influenced by several factors, for example, spatial organization of communities, local species abundances, and population aggregation (Morlon et al. 2008). Yet the underlying mechanisms generating observed distance decay in metacommunities remain poorly understood (Bolker 2004; Dunne 2006; Massol et al. 2011; Poisot et al. 2012).

In this article, we develop metacommunity models that differ in dispersal modes. The first model assumes that dispersal rates between patches are inversely proportional to the distance between patches. The second model assumes that dispersal is also influenced by the site size, in that immigration to larger sites is more probable than that to smaller sites. The third model assumes that immigration is negatively density dependent. The fourth model considers asymmetric dispersal when probability of dispersal from more centrally located sites to peripheral sites is more likely than dispersal in the opposite direction. We study these four population dispersal models either in homogeneous environments, where sites are evenly distributed across the landscape, or in heterogeneous environments, where site distribution is uneven. For these models we calculate the $\alpha$ (local), $\gamma$ (regional), and $\beta$ tritrophic richness. Using the empirical data describing a tritrophic metacommunity consisting of plants, aphids, and their parasitoids (Starý 2006), we fit our models to the empirical observations. We show that models with density-dependent dispersal in heterogeneous landscapes fit the empirical data better than models with density-independent dispersal. In particular, we show that models with densitydependent dispersal predict a critical distance between sites, beyond which similarity between local food webs sharply decreases. We also detect such a critical distance in the empirical data. Models with random dispersal do not predict such a distance threshold. Moreover, models with density-dependent dispersal predict a steeper decrease in $\beta$ tritrophic richness with distance when compared to models with random dispersal. Again, we show that such trends better agree with empirical observations. Our results suggest that nonrandom dispersal in heterogeneous landscapes is an important driver that shapes local and regional richness (i.e., $\alpha$ and $\gamma$ tritrophic richness, respectively) as well as the distance-decay relationship in multitrophic metacommunities (i.e., $\beta$ tritrophic richness).

\section{Plant-Aphid-Parasitoid Data}

The data that we analyze in this article describe tritrophic associations between 411 plant species, 267 aphid species, and 302 Hymenoptera parasitoid species (family Braconidae and subfamily Aphidiinae; Starý 2006). The data were collected at 302 sites in the Czech Republic between 1954 and 2004. Each site is characterized by two coordinates $\left(x_{1}, x_{2}\right)$ corresponding to its position on a grid overlaid on the map of the Czech Republic. The coordinates allow us to calculate the distance between two sites $\left(x_{1}\right.$, $\left.x_{2}\right)$ and $\left(y_{1}, y_{2}\right)$ as $d_{i j}=\left[A^{2}\left(x_{1}-y_{1}\right)^{2}+B^{2}\left(x_{2}-y_{2}\right)^{2}\right]^{1 / 2}$, where $A=12 \mathrm{~km}$ and $B=11.1 \mathrm{~km}$, representing the grid size of $12 \mathrm{~km} \times 11.1 \mathrm{~km}$. To capture the heterogeneity in site distribution across the landscape, we calculate for each site its total geographic distance from other sites (i.e., the sum of all distances between the focal site and all other sites). The corresponding distribution is used to classify landscapes as either homogeneous or heterogeneous. If distances between sites are independent, randomly distributed variables, the sum converges to a normal distribution. In what follows, we call landscapes with normally distributed total geographic distance "homogeneous," while those where the distribution significantly deviates from the normal distribution are called "heterogeneous" landscapes.

Sampling effort varied among sites: some were sampled many times, while others were sampled only once. In this article, we count each observed tritrophic chain at a given site only once; that is, multiple reports of the same chain at a given location are not accounted for. For 229 sites, the corresponding habitat type was reported, with some sites containing multiple habitat types (i.e., undergrowth, field, ruderal, road, hedge, park, steppe, meadow, pond, garden, forest, deciduous trees, gravel, orchard, town, greenhouse, alley, waste, rocky, and grassland; Starý 2006).

\section{$\alpha, \beta$, and $\gamma$ Richness of Tritrophic Chains}

To analyze tritrophic associations between plants, herbivores, and parasitoids, we adjust standard richness indexes. We define $\alpha$ tritrophic richness as the total number of different tritrophic chains in each sampled site. Two chains are different if they differ at least in one species. This measure is conceptually identical to $\alpha$ richness in ecolog- 
Table 1: Glossary of concepts

\begin{tabular}{ll}
\hline Concept & \multicolumn{1}{c}{ Explanation } \\
\hline Homogeneous landscape & $\begin{array}{r}\text { The sum of geographic distances from a focal site to all other sites is normally dis- } \\
\text { tributed; in our study we used the empirical mean distance between two sites, } \\
145 \mathrm{~km} \text {, and the empirical variance, } 50 \mathrm{~km} \text {, to generate the normal distance dis- } \\
\text { tribution in figure } 1 \text { (gray bars) } \\
\text { Distance distribution that deviates significantly from a normal distribution (fig. } 1, \\
\text { open bars) }\end{array}$ \\
$\begin{array}{l}\text { Dispersal probability between two sites is the same in both directions } \\
\text { Symmetric dispersal }\end{array}$ & $\begin{array}{l}\text { Dispersal probability between two sites depends on direction of dispersal } \\
\text { Asymmetric dispersal }\end{array}$ \\
$\begin{array}{l}\text { Colonizing a site is independent of species density } \\
\text { Negative density-dependent immigration }\end{array}$ & $\begin{array}{l}\text { Colonizing a site is a decreasing function of species density } \\
\text { Density-dependent mortality }\end{array}$ \\
$\begin{array}{l}\text { Probability to die is a function of species density } \\
\text { Density-dependent emigration }\end{array}$ & Probability to leave a site is a function of species density \\
\hline
\end{tabular}

ical communities, but it considers tritrophic associations instead of species. Thus, $\alpha$ tritrophic richness is a measure of the local richness in food chain configurations. Similarly, the regional $\gamma$ tritrophic richness is measured as the number of unique tritrophic chains observed across all sites.

Classical $\beta$ richness in community ecology measures similarity between sites by using pairwise comparison. This measure is useful when studying changes in species composition along ecological gradients. For two sites, $\beta$ richness compares species richness in each of the two sites with the number of species shared by the two sites (Magurran 2004; Poisot et al. 2012). If the data are a random collection of samples from a large region, then, in addition to the pairwise comparison, a multiple-site similarity measure is required to better capture the heterogeneity of habitats (Diserud and Ødegaard 2007). We calculate the twoand three-site Sørensen similarity indexes (Diserud and Ødegaard 2007) as $C_{\mathrm{S}}^{2}=2 c_{i j} /\left(u_{i}+u_{j}\right)$ and $C_{\mathrm{S}}^{3}=$ $(3 / 2)\left(c_{i j}+d_{i k}+e_{j k}-f_{i j k}\right) /\left(u_{i}+u_{j}+u_{k}\right)$, respectively, where $u_{i}, u_{j}$, and $u_{k}$ are the observed numbers of unique tritrophic chains in sites $i, j$, and $k$, respectively, and $c_{i j}, d_{i k}, e_{j k}$, and $f_{i j k}$ are the numbers of chains shared by $i$ and $j, i$ and $k$, $j$ and $k$, and $i, j$, and $k$ sites, respectively. Tritrophic chain similarity varies between 0 (completely dissimilar, no trophic chains in common) and 1 (completely similar, all trophic chains shared).

We are interested in the dependence of $\beta$ tritrophic similarity on distance between sites, that is, on the distance decay of similarity. Instead of calculating the mean $\beta$ tritrophic similarity index at a given distance, we focus on the maximum $\beta$ tritrophic similarity index, which better captures the decline in similarity between two (or three) sites (see "Results"). In the case of the two-site similarity index, we study this dependence as a function of distance between two sites, while in the case of the three-site similarity index, we plot this index as a function of the mean distance between three sites. We also compute the twosite habitat-specific $\beta$ tritrophic similarity index, which compares tritrophic chains in the same habitat type. For example, let us consider the situation where site $A$ contains habitat types $a, b$, and $c$ while site $B$ contains only habitat types $a$ and $c$. To compute the two-site $\beta$ tritrophic similarity index, we use all tritrophic chains found at both sites $A$ and $B$ independently of the habitat types. For the two-site habitat-specific $\beta$ tritrophic similarity index, we compare only those tritrophic chains in habitat $a$ only or those in habitat $c$ only.

We tested the robustness of tritrophic richness indexes to sampling effort by studying how they change when the number of sample sites increases (Polis 1991; Bersier et al. 1999). We did this by randomly sampling an increasing number of sites, taking into account all the unique tritrophic chains observed in each site, starting with 5, 10, $20,50,100,200$, and 300 sites from the original data set and calculating all three richness measures for each of these subsets. Because these measures are quantified as distributions, we describe each measure by using its mean value and its standard deviation. Random samplings were repeated 1,000 times to account for variability in tritrophic richness among sites.

\section{Models}

We consider a landscape consisting of $N$ sites. At each site, there is a food web consisting of resources $(R)$, consumers $(H)$, and parasitoids $(P)$. Collection of food chains at all sites represents a tritrophic metacommunity. To model spatiotemporal changes in population abundances, we need to define population and dispersal dynamics (the key terms are summarized in table 1). To simplify population dynamics, we assume that the number of individuals at each trophic level and site is fixed and equals the site environmental carrying capacity for the given trophic level. 
Thus, the overall number of individuals at each trophic level is fixed, but species composition changes over time because of replacement of each dead individual by a new individual from the same trophic level (but possibly of another species).

\section{Model 1: Density- and Site-Independent Dispersal Dynamics}

The first model assumes that dispersal rates are inversely proportional to the geographic distance between sites. Thus, dispersal to less distant sites is more likely than dispersal to more distant sites. This leads to the dispersal rate of species $k_{\phi}$ in metacommunity $\phi$ from site $j$ to site $i$ (where $\phi$ stands either for the resource $[R]$, the consumer $[H]$, or the parasitoid $[P]$ metacommunity),

$$
m_{i j}^{k_{\phi}}=\frac{m_{\phi}}{d_{i j}} .
$$

Here $d_{i j}$ is the geographical distance between sites $i$ and $j$, with $d_{i j}$ values obtained from the empirical data (see "Plant-Aphid-Parasitoid Data"), and $m_{\phi}$ is the intensity of emigration rate specific for each metacommunity $\phi$. Because dispersal from site $i$ to site $j$ is the same as that in the opposite direction $\left(m_{j i}^{k_{\phi}}=m_{i j}^{k_{\phi}}\right)$, model 1 represents symmetric and site- and density-independent dispersal.

\section{Model 2: Site-Dependent Dispersal Dynamics}

The second model assumes that dispersal rates depend not only on the geographic distance but also on the site environmental carrying capacity (denoted $J_{i}^{\phi}$ for site $i$ and metacommunity $\phi$ ). This modifies the dispersal rate from site $j$ to site $i$ of a species $k_{\phi}$ in model 1 , because now dispersing individuals have a higher probability of settling in a site that has a larger carrying capacity (which may be proportional to the site area). This leads to the following probability of settling in site $i$ :

$$
p_{i}^{\phi}=\frac{J_{i}^{\phi}}{\sum_{j=1}^{N} J_{j}^{\phi}} .
$$

Thus, the dispersal rate of species $k_{\phi}$ in metacommunity $\phi$ from site $j$ to site $i$ is

$$
m_{i j}^{k_{\phi}}=\frac{p_{i}^{\phi} m_{\phi}}{d_{i j}} .
$$

Dispersal model 2 represents symmetric and densityindependent dispersal. However, dispersal is site dependent, because immigration to larger sites (i.e., sites with a higher environmental carrying capacity) is more likely than that to smaller sites.

\section{Model 3: Density-Dependent Dispersal Dynamics}

The third model assumes that dispersal rates depend not only on the geographic distance but also on the number of individuals of the same species in the receiving site $i$. The dispersal rate of species $k_{\phi}$ in metacommunity $\phi$ from site $j$ to site $i$ is

$$
m_{i j}^{k_{\phi}}=\frac{J_{i}^{\phi}-N_{i}^{k_{\phi}}}{J_{i}^{\phi}} \frac{m_{\phi}}{d_{i j}},
$$

where $J_{i}^{\phi}$ has the same meaning as in model 2 and $N_{i}^{k_{\phi}}$ is the number of individuals of species $k_{\phi}$ of metacommunity $\phi$ that are already in site $i$. (We recall that the abundance of all individuals at a given trophic level is equal to the environmental carrying capacity of the site, i.e., $\sum_{k_{\phi}} N_{i}^{k_{\phi}}=J_{i}^{\phi}$.) This means that the immigration rate to a given site decreases as the number of conspecifics in the site increases. This may be the case where species within the same trophic level have different niches, so that they do not compete for their resources but there is competition for resources among individuals within the same species. Thus, this model considers intraspecific competition, but it neglects interspecific competition. We note that equation (4) is the same as equation (1) when no conspecifics are present in the receiving site $\left(N_{i}^{k_{\phi}}=0\right)$. Dispersal model 3 represents symmetric and density-dependent dispersal, because immigration depends negatively on the abundance of conspecifics at the receiving site.

\section{Model 4: Density-Dependent and Asymmetric Dispersal Dynamics}

While the previous three dispersal models assumed symmetric dispersal, model 4 considers asymmetric dispersal. It assumes that dispersal rates depend not only on the geographic distance and the number of conspecifics that are already in the receiving site but also on the spatial distribution of sites. One particular realization of such asymmetric dispersal from site $j$ to site $i$ of a species $k_{\phi}$ is

$$
m_{i j}^{k_{\phi}}= \begin{cases}\frac{J_{i}^{\phi}-N_{i}^{k_{\phi}}}{J_{i}^{\phi}} \frac{m_{\phi}}{d_{i j}} & \text { if } \sum_{k=1}^{N} d_{i k} \geq \sum_{k=1}^{N} d_{j k}, \\ 0 & \text { if } \sum_{k=1}^{N} d_{i k}<\sum_{k=1}^{N} d_{j k},\end{cases}
$$

where $N_{i}^{k_{\phi}}$ is the number of individuals of species $k_{\phi}$ of metacommunity $\phi$ that are already in site $i, m_{\phi}$ is the intensity of emigration rate specific for each metacommunity $\phi$, and $N$ is the number of sites. Formula (5) assumes that dispersal is unidirectional from sites with lower total geographic distance (where total geographic distance is defined as the sum of the distances between the focal site and all other sites) to sites with a higher total 
Table 2: Symbols and parameter values used in the text

\begin{tabular}{|c|c|}
\hline Symbol & Explanation \\
\hline$\phi$ & Resource $(R)$, consumer $(H)$, or parasitoid $(P)$ metacommunity \\
\hline$N_{i}^{k_{\phi}}$ & Abundance of species $k$ of metacommunity $\phi$ in site $i$ \\
\hline$S_{j}^{\phi}$ & Number of species in site $j$ of metacommunity $\phi$ \\
\hline$C_{\mathrm{S}}^{\mathrm{N}}$ & Sørensen similarity index for a number $N$ of sites \\
\hline$m_{i j}^{k_{\phi}}$ & Dispersal from site $j$ to site $i$ for species $k$ of metacommunity $\phi$ \\
\hline$m_{\phi}$ & Intensity of emigration rate of metacommunity $\phi$, randomly chosen from a uniform distribution $\mathcal{U}\left[7 \times 10^{-1}, 10^{-3}\right]$ \\
\hline$J_{i}^{\phi}$ & $\begin{array}{l}\text { Carrying capacity of site } i \text { of metacommunity } \phi \text {; for models } 1,3,4, J_{i}^{\phi}=1,000 \text {; for model } 2 \text {, it is chosen at random } \\
\text { from the normal distribution } \mathcal{N}[1,000,100]\end{array}$ \\
\hline$\nu_{\phi}$ & $\begin{array}{l}\text { Immigration rate from the regional species pool of metacommunity } \phi \text {, chosen at random from uniform distribution } \\
\mathcal{U}\left[10^{-2}, 10^{-4}\right]\end{array}$ \\
\hline$M_{i}^{k_{\phi}}$ & Density-dependent mortality of species $k$ in site $i$ and metacommunity $\phi$ \\
\hline$\mu_{\phi}^{k}$ & Natural mortality of species $k$ in metacommunity $\phi$ \\
\hline$\alpha^{k_{\phi} k_{\phi^{\prime}}^{\prime}}$ & $\begin{array}{l}\text { Mortality rate of species } k \text { of metacommunity } \phi \text { by species } k^{\prime} \text { of metacommunity } \varphi \text { due to the consumer or parasit- } \\
\text { oid species }\end{array}$ \\
\hline$\lambda_{\phi}$ & Local birth rate of metacommunity $\phi$ \\
\hline$d_{i j}$ & Geographical distance between site $i$ and $j$ \\
\hline$N$ & Number of sites (302) \\
\hline$C_{i}^{\mathrm{RH}}$ & Connectance in site $i$ for the resource-consumer food web with empirical values $[0.24,0.02]$ \\
\hline$C_{i}^{\mathrm{HP}}$ & Connectance in site $i$ for the consumer-parasitoid food web with empirical values $[0.24,0.02]$ \\
\hline
\end{tabular}

geographic distance. If we define core sites as those that have lower total geographic distance than peripheral sites, then formula (5) implies that individuals move from the core to the periphery of the landscape (Channell and Lomolino 2000).

\section{Dispersal Dynamics from the Regional Species Pool}

In addition to the dispersal dynamics between sites, new species can emerge with very low probability $\left(\nu_{\phi}\right.$ for metacommunity $\phi$ ) from the regional species pool. We consider an extremely diverse regional species pool at each trophic level, containing an infinite number of species. Thus, we assume that every immigration event introduces a new species. Immigration of a new species corresponds to speciation in the context of metacommunity models (Vanpeteghem and Haegeman 2010).

\section{Multitrophic Metacommunity Dynamics}

Here, we describe population dispersal dynamics. At each time step, one site for each metacommunity $\phi$ is chosen with probability $p_{i}^{\phi}$. For dispersal models 1,3 , and 4 , this selection is random (i.e., $p_{i}^{\phi}=1 / N$ where $N$ is the number of sites), while model 2 assumes probability given by equation (2). In each of the three chosen sites, a single death event occurs in each of the three metacommunities. This death event is compensated for by recruitment of a new individual, so that the total number of individuals does not change during simulations. However, as the new individual can be of another species, the species composition changes over time. The recruitment is due to either local reproduction (birth), immigration from another site, or immigration from the regional species pool. The key parameters used throughout the article are summarized in table 2, and the models are described in detail in appendix A (apps. A-D available online).

\section{Simulations and Parameter Estimation}

To infer the dispersal mode that fits the empirical data best, we numerically simulate the four population dispersal models in either homogeneous or heterogeneous landscapes. For each of eight possible combinations of dispersal modes, we run 100,000 replicates. We assume that, initially, each trophic level contains only one species. For each simulation run (i.e., a replicate), the number of generations is chosen at random from a uniform distribution $\mathcal{U}[100$, 30,000]. The number of individuals per site (i.e., the site environmental carrying capacity $J_{i}^{\phi}$ ) within each trophic level $\phi$ is set to 1,000 in models 1,3 , and 4. For model 2 , the number of individuals per site varies across sites within each replicate, and these numbers are chosen from a normal distribution $\mathcal{N}[1,000,100]$, where 1,000 is the mean and 100 is the variance. We set all mortality rates equal to 1 (i.e., the natural mortality rate for plants $\left[\mu^{k_{\mathrm{R}}}\right]$ and parasitoids $\left[\mu^{k_{\mathrm{p}}}\right]$ and the mortality rate of aphids due to parasitism $\left.\left[\alpha^{k_{\mathrm{H} k \mathrm{k}}}\right]\right)$. Rates of immigration from the regional species pool, $\nu_{\phi}$, and emigration rates specific for each metacommunity, $m_{\phi}$, are chosen at random from the uniform distributions $\mathcal{U}\left[10^{-4}, 10^{-2}\right]$ and $\mathcal{U}\left[10^{-3}, 7 \times\right.$ $10^{-1}$, respectively. Local birth rates for each metacom- 
munity $\lambda_{\phi}=1-\nu_{\phi}-m_{\phi}$, so that a new individual replacing the dead individual appears with certainty.

In our simulations, we check whether the resulting food web at the end of the simulation fits the empirical connectance at each site. Connectances in each site $i$ for the resource-host and the host-parasitoid are defined as $C_{i}^{\mathrm{RH}}=\left(L_{i}^{\mathrm{RH}}\right) /\left(S_{i}^{\mathrm{R}} S_{i}^{\mathrm{H}}\right)$ and $C_{i}^{\mathrm{HP}}=\left(L_{i}^{\mathrm{HP}}\right) /\left(S_{i}^{\mathrm{H}} S_{i}^{\mathrm{P}}\right)$, respectively. Here, $L_{i}^{\mathrm{RH}}, L_{i}^{\mathrm{HP}}, S_{i}^{\mathrm{R}}, S_{i}^{\mathrm{H}}$, and $S_{i}^{\mathrm{P}}$ are the number of interactions between plants and aphids and between aphids and parasitoids and the number of plant, aphids, and parasitoid species, respectively. After each simulation run (i.e., a replicate), we check whether the simulated connectance in each site $i$ between plants and aphids and between aphids and parasitoids is in the empirical range of the minimum and maximum observed connectance values, $0.02-0.24$. A replicate is removed if any of the sites is outside the empirical range. About $10 \%$ of replicates were removed.

For each simulation run (i.e., for each specific parameter choice), we calculate the $\alpha$ and $\gamma$ tritrophic richness and compare them with the empirical values. We use a tolerance $(\varepsilon)$ rejection algorithm for model choice within an approximate Bayesian computation framework to do these comparisons (Grelaud et al. 2009; Beaumont 2010). This means that for each simulation run, we calculate the "misfit" (for definition, see eq. [B1], in app. B), which measures the difference between the observed and the predicted number of tritrophic chains. A simulation run is assumed to provide a good fit between the model and the empirical data if the misfit is close to 0 . As the misfits are negative, this corresponds to misfits that are above the $\varepsilon$ tolerance threshold (see app. B and fig. B1; figs. B1, C1, D1, and D2 available online). This means that such simulation runs predict $\alpha$ and $\gamma$ tritrophic richness values that are sufficiently close to the empirical values. Model parameters that correspond to simulations that meet the $\varepsilon$ tolerance threshold are called the "best-fit" parameters.

\section{Results}

The distribution of sampled sites (fig. 1, open bars) is significantly different from a normal distribution (fig. 1, gray bars) with the empirical mean $(145 \mathrm{~km})$ and variance (50 km; $P<.0001$, Kolmogorov-Smirnov test). In our terminology, this means that the sampled sites form a spatially heterogeneous landscape.

\section{$\alpha$ and $\gamma$ Tritrophic Richness}

The empirical data contain 4,966 tritrophic chains across all sites. Observed $\gamma$ tritrophic richness is 1,304 unique tritrophic chains. Our analysis shows that sampling effort was not sufficient to estimate $\gamma$ tritrophic richness, because

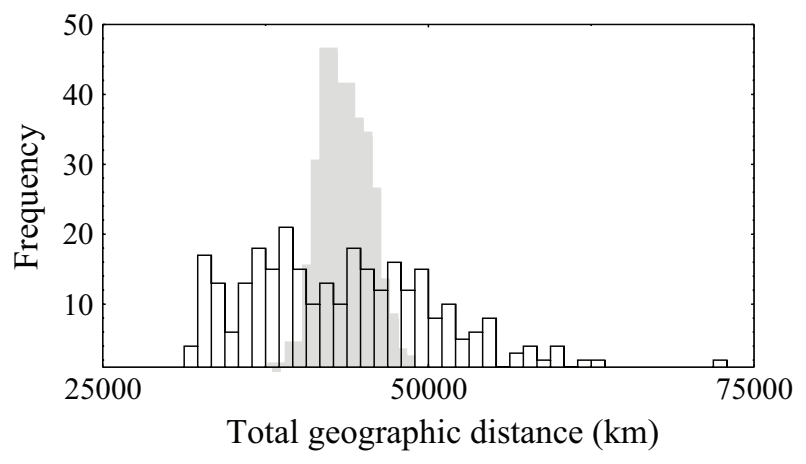

Figure 1: Distribution of total geographic distances for all sites. The total geographic distance for a given site is the sum of the distances between this focal site and all other sites. The distribution for sampled sites is shown as open bars, while the normal distribution with the same mean $(145 \mathrm{~km})$ and variance $(50 \mathrm{~km})$ is shown in gray. This figure shows that the empirical site distribution corresponds to a heterogeneous landscape, as it deviates significantly from the normal site distribution.

the richness does not level off (app. C; fig. C1A, C1B). In other words, the observed $\gamma$ tritrophic richness underestimates the real richness. At many sites, only one tritrophic chain was observed, and fewer than $16 \%$ of sites hosted more than 10 different chains. The mean number of tritrophic chains per site, the mean $\alpha$ tritrophic richness, equals 16.4 , and the spatial variation in the number of tritrophic chains equals 6,078 . The sampling effort to estimate $\alpha$ tritrophic richness was sufficient because $\alpha$ tritrophic richness saturates after sampling of 302 sites (fig. $\mathrm{C} 1 C, \mathrm{C} 1 D)$.

Using empirical data, we estimated parameters of our four models (assuming the empirical heterogeneous site distribution) that best predict the local and regional tritrophic richness (i.e., $\alpha$ and $\gamma$ tritrophic richness, respectively). Density-dependent dispersal models (models 3 and 4) predict the local and regional richness of chains equally best. In tables 3 and 4, this corresponds to Bayes factors greater than 2 (see also app. B; table B1). Model 4, with preferential dispersal from more centrally located sites to peripheral sites, does not provide a better fit to empirical data when compared to model 3 , with symmetric dispersal (Bayes factors values $<0.5$; tables 3,4 ).

These results are graphically presented in figure $2(\alpha$ tritrophic richness in the left-hand and middle columns and $\gamma$ tritrophic richness in the right-hand column). Each dot corresponds to one simulation with randomly chosen parameters from given distributions (see "Simulations and Parameter Estimation"). For each simulation, we calculated the corresponding mean and variance of $\alpha$ tritrophic richness and $\gamma$ tritrophic richness for three different ranges of emigration rates (orange, red, and black dots represent 
Table 3: Comparison of the four models $\left(m_{1}-m_{4}\right)$ to predict $\alpha$ tritrophic richness

\begin{tabular}{lccc}
\hline & \multicolumn{3}{c}{ Bayes factors $\left(\log \left(\mathrm{BF}_{m_{k} / m_{j}}\right)\right.$} \\
\cline { 2 - 4 } Tolerance $(\varepsilon)$, model & Model $4(k=4)$ & Model 3 $(k=3)$ & Model 2 $(k=2)$ \\
\hline$q_{1 \%}:$ & & 4.3 & 1 \\
Model $1(j=1)$ & 4.2 & 3.3 & .9 \\
Model 2 $(j=2)$ & 3.2 & & \\
Model 3 $(j=3)$ & .1 & 4.4 & 1.2 \\
$q_{3 \%}$ : & 5.3 & 5.4 & \\
Model 1 $(j=1)$ & 4.3 & & \\
Model 2 $(j=2)$ & .04 & 4.9 & \\
Model 3 $(j=3)$ & & 5.8 & \\
$q_{5 \%}$ : & 5.9 & & \\
Model $1(j=1)$ & 4.9 & & \\
Model 2 $(j=2)$ & .02 & & \\
Model 3 $(j=3)$ & &
\end{tabular}

Note: Comparisons of the four models are based on Bayes factors $\left(\mathrm{BF}_{m_{b} / m_{j}}\right)$ according to the Jeffreys scale (decisive: $\log \left(\mathrm{BF}_{m_{k} / m_{j}}\right)>2$; strong: $1<\log \left(\mathrm{BF}_{m_{k} / m_{j}}\right)<2$; substantial: $0.5<\log \left(\mathrm{BF}_{m_{k} / m_{j}}\right)<1$; weak: $\left.0<\log \left(\mathrm{BF}_{m_{k} / m_{m}}\right)<0.5\right)$. The four models are $m_{1}$, density- and site-independent dispersal dynamics; $m_{2}$, site-dependent dispersal dynamics; $m_{3}$, density-dependent dispersal dynamics; and $m_{4}$, densitydependent and asymmetric dispersal dynamics. The three tolerance threshold values, $\varepsilon$, correspond to the $1 \%, 3 \%$, and $5 \%$ upper quantiles of the distribution of misfits.

low $\left[m_{\phi} \in\left[10^{-3}, 10^{-1}\right]\right]$, medium $\left[m_{\phi} \in\left[10^{-1}, 2 \times 10^{-1}\right]\right]$, and high $\left[m_{\phi} \in\left[2 \times 10^{-1}, 7 \times 10^{-1}\right]\right]$ intensity of emigration rate, respectively). Then, we calculated the fit (app. B) between the empirical data and the simulation. For each simulation run, we calculated the misfit (defined by eq. [B1]) between the predicted and observed numbers of food chains in the metacommunity. Then, we plotted the distribution of these misfits (see fig. B1) and calculated the misfit value that corresponds to the $1 \%$ quantile (see the horizontal lines in fig. 2 and the rightmost vertical line in fig. B1). This quantile corresponds to tolerance $\varepsilon_{1}=$ $-1,338.5$. The empirically observed mean (16.4) and variance $(6,078)$ of $\alpha$ richness and $\gamma$ tritrophic richness $(1,304)$ are plotted as vertical lines in figure 2 . Dots that are above the horizontal line correspond to parameters that fit the observed data best. Models 3 and 4, with fitted parameters, predict the empirical mean $\alpha$ richness well (i.e., observed 16.4 vs. predicted $13.6 \pm 6$; fig. 2, left) for low to medium dispersal rates. However, all models underestimate the empirical spatial variation of the number of tritrophic chains per site (fig. 2, middle; the observed variance is shown as the vertical line). Only when dispersal intensity is high do models 3 and 4 predict the variance correctly, but in this case the fit between predicted and observed numbers of tritrophic chains is poor (fig. $2 C$, $2 D$, left).

The predicted $\gamma$ tritrophic richness for density-dependent models 3 and 4 that fits the observed food web best does not show a significant difference from the observed $\gamma$ tritrophic richness (table 4 and the right-hand column of fig. $2 C, 2 D)$. Thus, preferential dispersal from core sites to peripheral sites did not significantly improve results of nonpreferential dispersal. Similarly to the $\alpha$ tritrophic richness, most tritrophic chains were observed only once across all sampled sites, while only about $1 \%$ of the observed chains were observed in more than 10 locations. However, these predictions should be interpreted with caution, because, as we have already mentioned, the sampling effort was insufficient to estimate $\gamma$ tritrophic richness (fig. $\mathrm{C} 1 A, \mathrm{C} 1 B)$.

\section{$\beta$ Tritrophic Richness}

To study $\beta$ tritrophic richness, we plot the maximum site similarity in the true tritrophic plant-aphid-parasitoid data as a function of geographic distance (fig. 3, dots). For a given distance between two or three sites, this figure shows the maximum number of shared tritrophic chains among two (fig. $3 A$, top) or three sites (fig. $3 B$, top; $X$-axes represents the mean distance between the three sites). While figures $3 A$ and $3 B$ do not consider habitat types within a given site, figure $3 C$ compares only tritrophic chains within the same habitat type. The solid lines (together with confidence intervals, shown by dotted lines) in figure 3 correspond to the maximum similarity values predicted by model 3, with density-dependent and symmetric dispersal. We observe that most empirical values (shown as dots) fit within the confidence intervals for the two-site (fig. $3 A$, top) and the three-site (fig. $3 B$, top) comparisons but that the fit is not as good once only the same habitat types are compared (fig. 3C, top). In the latter case, $23 \%$ of the 
Table 4: Comparison of the four models $\left(m_{1}-m_{4}\right)$ to predict $\gamma$ tritrophic richness

\begin{tabular}{lccc}
\hline & \multicolumn{3}{c}{ Bayes factors $\left(\log \left(\mathrm{BF}_{m_{k} / m_{j}}\right)\right.$} \\
\cline { 2 - 4 } Tolerance $(\varepsilon)$, model & Model $4(k=4)$ & Model $3(k=3)$ & Model 2 $(k=2)$ \\
\hline$q_{1 \%}:$ & & 2.4 & .1 \\
Model $1(j=1)$ & 2.4 & 2.3 & .6 \\
Model 2 $(j=2)$ & 2.5 & & \\
Model 3 $(j=3)$ & .1 & 2.8 & .6 \\
$q_{3 \%}:$ & & 3.4 & \\
Model $1(j=1)$ & 3.5 & & \\
Model 2 $(j=2)$ & 2.9 & 3.3 \\
Model 3 $(j=3)$ & .16 & 3.9 \\
$q_{5 \%}:$ & & \\
Model $1(j=1)$ & 4.1 &
\end{tabular}

empirically observed data deviate significantly from the predicted values.

Figure 3 shows that model 3 predicts a lower decrease in similarity at short distances (see the slope of the solid line for distances approximately between 0 and $200 \mathrm{~km}$ ), followed by a steeper decrease (approximately between 200 and $350 \mathrm{~km}$ ). Beyond $350 \mathrm{~km}$, the two- and three-site tritrophic chain similarity values are near 0 (fig. $3 A, 3 B$ ). However, when the same habitat types are compared, even sites whose distance is $600 \mathrm{~km}$ can be highly similar (fig. $3 C$ ). This means that tritrophic chains are specific for each habitat type. To test whether there is a critical distance threshold beyond which the similarity between sites sharply decreases in empirical data, we calculate the variance of site similarity as a function of geographical distance. We note that small variance in similarity index at a given distance means that this index does not change very much for sites separated by this distance. At distances where this index changes sharply, the variance should be maximal. The variance in site similarity in empirical data peaks at approximately $350 \mathrm{~km}$ for the two- and threesite similarity indexes (fig. 3, bottom).

The same pattern is predicted by model 3 when applied to a heterogeneous landscape (fig. D2D). The predicted distance decay of variance of similarity shows no peak across all the geographic distances for model 1, with density-independent dispersal, either in homogeneous (fig. D2C, red dots) or in heterogeneous (fig. D2C, black dots) landscapes. Results for models 2 and 4 are similar to those for models 1 and 3, respectively, and are not shown here. These results lead to the following two postulates: (1) individuals disperse preferentially to sites where conspecifics are rare (model 3), and (2) asymmetric dispersal (model 4) does not improve the fit between observed and simulated data when compared to symmetric dispersal (model
3) between sites. The effect of empirical sampling effort on regional similarity, the $\beta$ tritrophic richness, levels off after approximately 100 sites were sampled (fig. $\mathrm{C} 1 E, \mathrm{C} 1 F$ ). This suggests that the sampling effort when collecting empirical data for plant-aphid-parasitoid interactions was adequate to estimate $\beta$ tritrophic richness.

\section{Estimated Parameter Values}

From model 3 we estimated metacommunity-specific emigration rates and the immigration rates from the regional species pool that best predict the empirical tritrophic richness at local and regional scales. These estimates were obtained from all simulation runs with misfits above the $3 \%$ quantile of the misfit distribution (fig. B1). Neither emigration rates $(P>.1$; Kolmogorov-Smirnov test for all pairwise comparisons with median value $\left.m_{\mathrm{R}} \sim m_{\mathrm{H}} \sim m_{\mathrm{P}} \sim 0.05\right)$ nor immigration rates from the regional species pool $(P>$ .1; Kolmogorov-Smirnov test with median value $\nu_{\mathrm{R}} \sim$ $\left.\nu_{\mathrm{H}} \sim \nu_{\mathrm{P}} \sim 0.0045\right)$ show significant differences across trophic levels. These estimated values imply that around $5 \%$ of individuals disperse per site and per generation within the sampled area. Within the range of dispersal rates considered in this article, the estimated dispersal rates belong to the range of low dispersal rates represented by orange dots in figure 2 (which represent a low intensity of emigration rates, $\left.m_{\phi} \in\left[10^{-3}, 10^{-1}\right]\right)$.

\section{Discussion}

In this article, we study mechanisms generating $\alpha, \beta$, and $\gamma$ tritrophic richness observed in an empirical metacommunity consisting of plants, aphids, and their parasitoids. We compare these empirical observations with predictions of a series of models that differ in dispersal (density in- 
A) Model 1
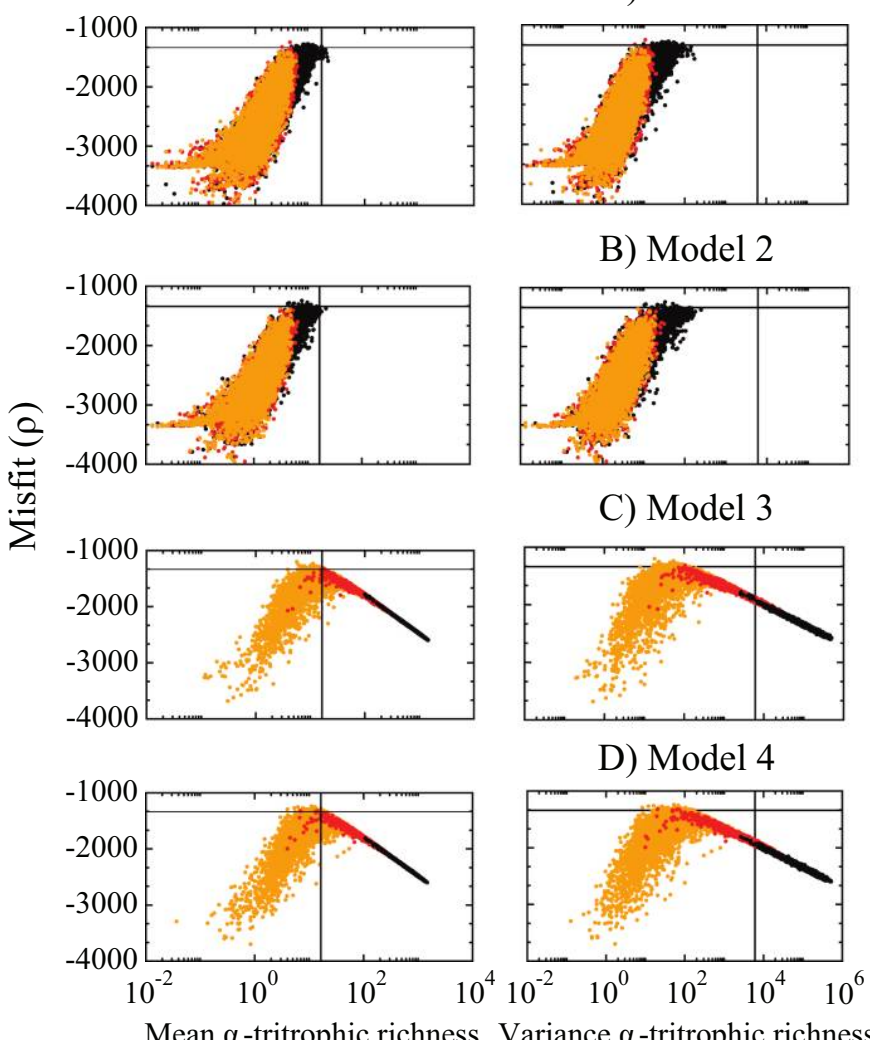

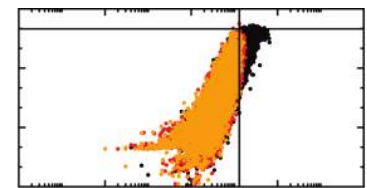

B) Model 2

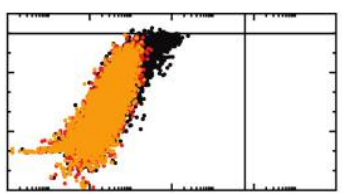

C) Model 3
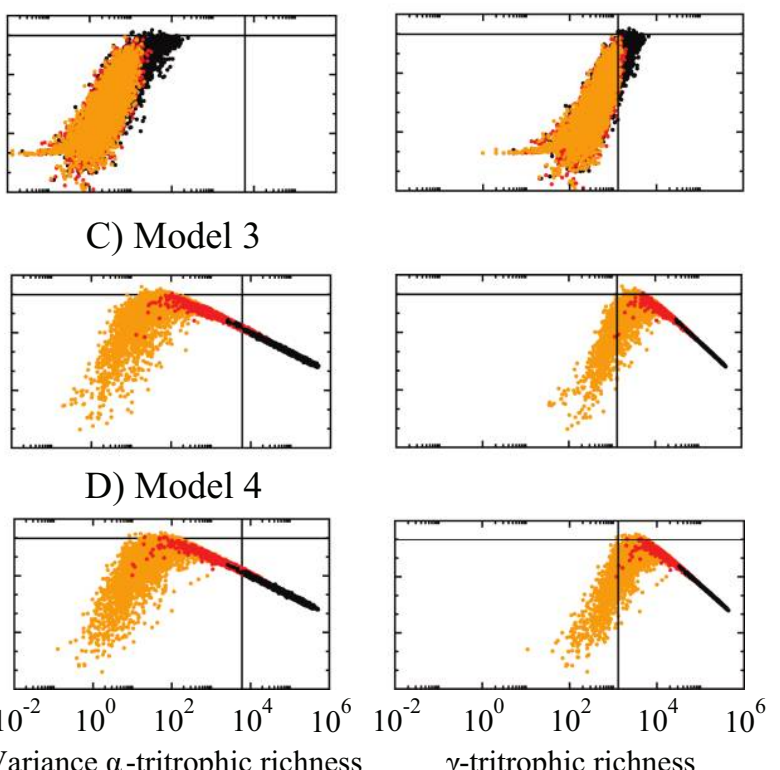

Figure 2: Fit between model and data for $\alpha$ and $\gamma$ tritrophic richness. Each dot represents a single simulation run of the corresponding model, 1-4. The $Y$-coordinate represents the misfit ( $\rho$; eq. [B1], available online) between the simulated food web and the empirical metacommunity. The $X$-coordinate represents the mean of $\alpha$ tritrophic richness of the simulated metacommunity (left), the variance of $\alpha$ tritrophic richness (middle), or $\gamma$ tritrophic richness (right). The variance of $\alpha$ tritrophic richness predicted by simulations is shown in the middle column. A horizontal line represents the upper $1 \%$ quantile of the misfits (with the corresponding tolerance value $\varepsilon_{1}=-1,338.5$ ) after 100,000 replicates for each model. The vertical lines in the left- and right-hand panels represent the mean $\alpha$ tritrophic richness (16.4, left) and the $\gamma$ tritrophic richness $(1,304$, right $)$. The vertical line in the middle panels represents the empirical variance of the $\alpha$ tritrophic richness. Orange, red, and black dots represent low $\left(m_{\phi} \in\left[10^{-3}, 10^{-1}\right]\right)$, medium $\left(m_{\phi} \in\left[10^{-1}, 2 \times 10^{-1}\right]\right)$, and high $\left(m_{\phi} \in\left[2 \times 10^{-1}\right.\right.$, $\left.7 \times 10^{-1}\right]$ ) intensity of emigration rate, respectively.

dependent vs. density dependent; symmetric vs. asymmetric) and spatial distribution of sampled sites (homogeneous vs. heterogeneous landscapes). Among these models, those with density-dependent dispersal fit $\alpha, \beta$, and $\gamma$ tritrophic richness observed in empirical data better than those with density-independent dispersal (cf. models 3 and 4 , with density-dependent dispersal, and models 1 and 2, with density-independent dispersal, in fig. 2). Our study confirms that nonrandom dispersal has a strong effect on empirical patterns of food webs connected by dispersal (Shurin 2001; Holt and Hoopes 2005; Economo and Keitt 2008; Massol et al. 2011; Carrara et al. 2012). Our analysis shows that the results for symmetric dispersal are as good as those for asymmetric dispersal (cf. results for model 3, with symmetric dispersal, and those for model 4 , with asymmetric dispersal, in tables 3 and 4). Our results suggest that models with density-dependent dispersal in heterogeneous landscapes that mimic the empirical site distribution capture the observed pattern of the distance decay of similarity. Using the two- and three-site Sørensen similarity indexes, we calculate the maximum number of shared tritrophic chains between two or three sites at a given distance interval (solid lines in fig. 3). Taking the maximum instead of the mean number of shared chains (i.e., the mean number of shared tritrophic chains at the given distance interval) captures much better the decrease in similarity with distance (cf. fig. 3 and fig. D1).

Previous studies suggest that strong dispersal limitation induces a steep decay in community similarity (Nekola and Peter 1999; Morlon et al. 2008; McClain et al. 2012). Our results show that in addition to the strong dispersal limitation in each metacommunity, nonrandom dispersal 
A
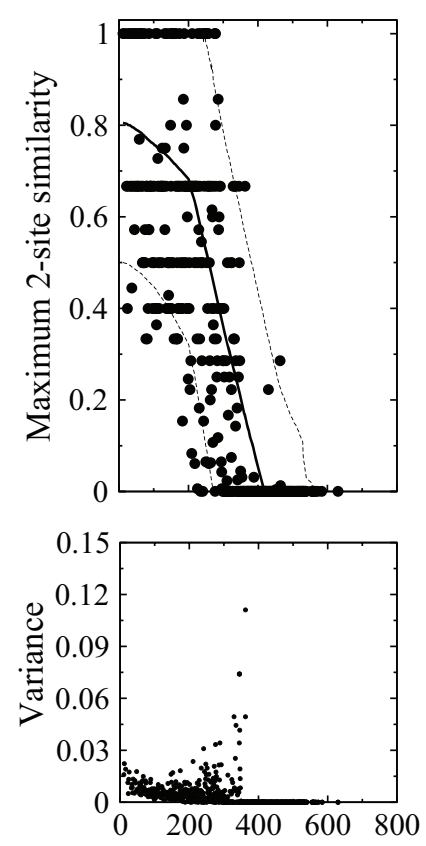

$\mathrm{B}$
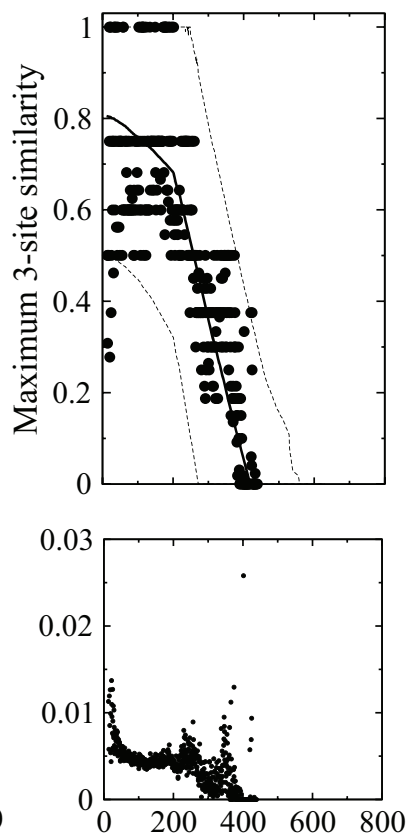

$\mathrm{C}$
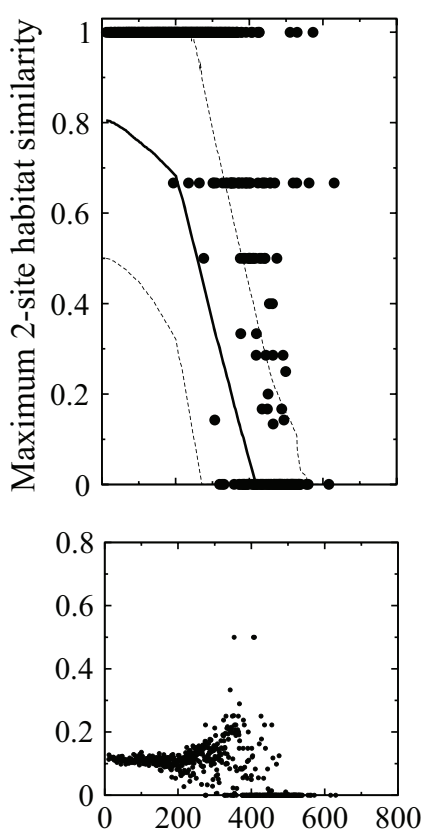

Geographic distance $(\mathrm{km})$

Figure 3: Empirical distance decay of maximum similarity (top) and of variance of similarity (bottom) for two-site $\beta$ tritrophic similarity $(A)$, three-site $\beta$ tritrophic similarity $(B)$, and two-site habitat-specific $\beta$ tritrophic similarity $(C)$. We use all tritrophic chains found in each pair (trio) of sites to compute the two-site (three-site) $\beta$ tritrophic similarity in $A(B)$. For the two-site habitat-specific $\beta$ tritrophic similarity in $C$, we compare only those tritrophic chains within a given habitat type. Solid lines represent the means of the maximum similarity predicted by model 3, with symmetric and density-dependent dispersal dynamics, and dotted lines the confidence intervals.

in heterogeneous landscapes leads to a critical distance threshold beyond which the maximum site similarity decreases much faster (this critical distance in fig. 3 is roughly $200 \mathrm{~km}$; see the sharp corner of the solid line). This threshold is also characterized by a peak in the variance of the distance-decay similarity in the empirical data (bottom panels in fig. 3 show such a peak in the empirical data, while fig. D2D shows a peak in simulated metacommunities). Neither models with density-independent dispersal nor models that assume homogeneous landscapes predict such a critical threshold (fig. D2).

We also showed that $\beta$ tritrophic richness is strongly influenced by habitat similarity. We contrasted distance decay in site similarity without (fig. $3 A$ ) and with (fig. $3 C$ ) accounting for habitat similarity. In the first case, we compared all tritrophic chains in all habitats within a given site, while in the latter case we compared only tritrophic chains in the same habitat. Altogether, there were 20 different habitat types (see "Plant-Aphid-Parasitoid Data"). In our empirical data, beyond $350 \mathrm{~km}$, the two-site (fig. $3 A$ ) and three-site (fig. $3 B$ ) tritrophic chain similarity was near 0 for most pairwise comparisons. However, after hab- itat similarity was accounted for, even sites whose distance is $600 \mathrm{~km}$ could be highly similar (fig. 3C). Thus, species preference for specific habitat types is an important factor influencing $\beta$ tritrophic richness. These results suggest that preferential habitat choice predicts more even distribution of tritrophic chains with geographic distance. This suggests that adding preferential habitat choice to our models could improve the fit with empirical observations (Mouquet and Loreau 2003; Haegeman and Loreau 2014). We remark, however, that up to $77 \%$ of the empirically observed tritrophic chains are still within the limits predicted by our models that do not consider habitat preferences (dotted lines in fig. 3C).

In our analyses, most of the variation in tritrophic richness remains unexplained (fig. 2, middle). Only when dispersal is high do models 3 and 4 predict the observed variance in tritrophic richness correctly (fig. $2 C, 2 D$, middle, black dots), but in this case the predicted $\alpha$ tritrophic richness strongly deviates from the empirical value (fig. $2 C, 2 D$, left, black dots). For metacommunities with resource-consumer-predator dynamics in each patch, an increase in the dispersal rates causes strong and synchro- 
nous food web fluctuations (Gouhier et al. 2010; but $\sec \rightarrow$ Koelle and Vandermeer 2005). Synchrony in local population fluctuations increases variability in the $\alpha$ richness in the metacommunity (Thibaut and Connolly 2013; Wang and Loreau 2014). In our models, increasing correlation in dispersal between different trophic levels can lead tc $\rightarrow$ such synchronous fluctuations and thus to better predictions of the observed empirical variability of $\alpha$ tritrophic richness in our multitrophic metacommunity.

A theory of food webs in spatial landscapes is now entering a predictive stage (McCann et al. 2005; Cummin et al. 2010; Dale and Fortin 2010; Gouhier et al. 2010; Gravel et al. 2011; Massol et al. 2011; Poisot et al. 2012; Haegeman and Loreau 2014). A central challenge in this predictive era is to develop methods to infer the processe driving patterns of food webs across broad geographic regions from empirical observations. This may help us to understand the drivers that shape biodiversity patterns and the biogeography of food webs. This article shows that density-dependent and symmetric dispersal in heterogeneous landscapes may add up to other factors to predic $\rightarrow$ the empirical patterns in local food webs (i.e., $\alpha$ tritrophic richness) and to connect the local patterns to turnover rates across geographically distant food webs (i.e., $\beta$ and $\gamma$ tritrophic richness).

Data reported in this article are deposited in the Dryad Data Repository: http://doi.org/10.5061/dryad.06bb5 (Me. lián et al. 2015).
Araújo, M. B., and M. Luoto. 2007. The importance of biotic interactions for modelling species distributions under climate change. Global Ecology and Biogeography 16:743-753.

Beaumont, M. A. 2010. Approximate Bayesian computation in evolution and ecology. Annual Review of Ecology, Evolution, and Systematics 41:379-406.

Bersier, L.-F., P. Dixon, and G. Sugihara. 1999. Scale-invariant or scale-dependent behavior of the link density property in food webs. American Naturalist 153:676-682.

Bolker, B. 2004. Continuous-space models for population dynamics. Pages 45-69 in I. Hanski and O. E. Gaggioti, eds. Ecology, genetics, and evolution of metapopulations. Elsevier, San Diego, CA.

Boulangeat, I., D. Gravel, and W. Thuiller. 2012. Accounting for dispersal and biotic interactions to disentangle the drivers of species distributions and their abundances. Ecology Letters 15:584593.

Briggs, C. J., and M. F. Hoopes. 2004. Stabilizing effects in spatial parasitoid-host and predator-prey models: a review. Theoretical Population Biology 65:299-315.

Carrara, F., F. Altermatt, I. Rodriguez-Iturbe, and A. Rinaldo. 2012. Dendritic connectivity controls biodiversity patterns in experimental metacommunities. Proceedings of the National Academy of Sciences of the USA 109:5761-5766.

Channell, R., and M. V. Lomolino. 2000. Dynamic biogeography and conservation of endangered species. Nature 403:84-86.

Cumming, G. S., O. Bodin, H. Ernstson, and T. Elmqvist. 2010. Network analysis in conservation biogeography: challenges and opportunities. Diversity and Distributions 16:414-425.

$\rightarrow$ Dale, M., and M.-J. Fortin. 2010. From graphs to spatial graphs. Annual Review of Ecology, Evolution, and Systematics 41:21-38.

$\rightarrow$ Diserud, O. H., and F. Ødegaard. 2007. A multiple-site similarity measure. Biology Letters 3:20-22.

Dunne, J. A. 2006. The network structure of food webs. Pages 2786 in M. Pascual and J. A. Dunne, eds. Ecological networks: linking structure to dynamics in food webs. Oxford University Press, Oxford.

\section{Acknowledgments}

We thank J. Astegiano, F. Briand, N. Holland, and three anonymous reviewers for valuable suggestions that greatly improved the manuscript. C.J.M. was supported by the Swiss National Science Foundation project 31003A-144162 and partially supported by a postdoctoral fellowship at the National Center for Ecological Analysis and Synthesis, a center funded by the National Science Foundation (gran DEB-0553768); the University of California, Santa Barbara; and the State of California. C.J.M. also acknowledges the support by Microsoft Research, Cambridge, Unitec Kingdom. P.S. and V.K. acknowledge support by the Institute of Entomology (RVO:60077344). F.D.L. is a postdoctoral research fellow from the Fund for Scientific Research-Flanders (Fonds Wetenschappelijk OnderzoekVlaanderen).

\section{Literature Cited}

Amarasekare, P. 2008. Spatial dynamics of foodwebs. Annual Review of Ecology, Evolution, and Systematics 39:479-500.
Economo, E. P., and T. Keitt. 2008. Species diversity in neutral metacommunities: a network approach. Ecology Letters 11:52-62.

Gouhier, T. C., F. Guichard, and A. Gonzalez. 2010. Synchrony and stability of food webs in metacommunities. American Naturalist 175:E16-E34.

Gravel, D., F. Massol, E. Canard, D. Mouillot, and N. Mouquet. 2011. Trophic theory of island biogeography. Ecology Letters 14:10101016.

Grelaud, A., C. P. Robert, J.-M. Marin, F. Rodolphe, and J.-F. Taly. 2009. ABC likelihood-free methods for model choice in Gibbs random fields. Bayesian Analysis 4:317-336.

Haegeman, B., and M. Loreau. 2014. General relationships between consumer dispersal, resource dispersal and metacommunity di-

Hanski, I. 1999. Habitat connectivity, habitat continuity, and metapopulations in dynamic landscapes. Oikos 87:209-219.

Holt, R. D., and M. Hoopes. 2005. Food web dynamics in a metacommunity context: modules and beyond. Pages 68-93 in M. Holyoak, M. A. Leibold, and R. Holt, eds. Metacommunities: spatial dynamics and ecological communities. University of Chicago Press, Chicago.

$\rightarrow$ Huffaker, C. B. 1958. Experimental studies on predation: dispersion factors and predator-prey oscillations. Hilgardia 27:795-834.

$\rightarrow$ Kissling, W. D., C. F. Dormann, J. Groeneveld, T. Hickler, I. Kühn, versity. Ecology Letters 17:175-184. 
G. J. McInerny, J. M. Montoya, et al. 2012. Towards novel approaches to modelling biotic interactions in multispecies assemblages at large spatial extents. Journal of Biogeography 39:21632178.

$\rightarrow$ Koelle, K., and J. Vandermeer. 2005. Dispersal-induced desynchron $\rightarrow$ ization: from metapopulations to metacommunities. Ecology Letters 8:167-175.

Křivan, V. 2008. Predator-prey models. Pages 2929-2940 in S. E $\rightarrow$ Jørgensen and B. D. Fath, eds. Population dynamics. Vol. 4 of Encyclopedia of ecology. Elsevier, Oxford.

$\rightarrow-$.2014. Competition in di- and tri-trophic food web modules Journal of Theoretical Biology 343:127-137.

$\rightarrow$ Leibold, M. A., M. Holyoak, N. Mouquet, P. Amarasekare, J. M. Chase, and M. F. Hoopes. 2004. The metacommunity concept: $\rightarrow$ framework for multi-scale community ecology. Ecology Letters 7 : 601-613.

$\rightarrow$ Levins, R. 1962. Theory of fitnesses in a heterogeneous environment I. The fitness set and adaptive function. American Naturalist 96: 361-378.

Magurran, A. 2004. Measuring biological diversity. Blackwell, Oxford.

$\rightarrow$ Massol, F., D. Gravel, N. Mouquet, M. W. Cadotte, T. Fukami, and M. A. Leibold. 2011. Linking community and ecosystem dynamics through spatial ecology. Ecology Letters 14:313-323.

$\rightarrow$ McCann, K. S., J. B. Rasmussen, and J. Umbanhowar. 2005. Thı $\rightarrow$ dynamics of spatially coupled food webs. Ecology Letters 8:513523.

$\rightarrow$ McClain, C. R., J. C. Stegen, and A. H. Hurlbert. 2012. Dispersal, environmental niches and oceanic-scale turnover in deep-sea bivalves. Proceedings of the Royal Society B: Biological Sciences 279: 1993-2002.

Melián, C. J., V. Křivan, F. Altermatt, P. Starý, L. Pellissier, and F De Laender. 2015. Data from: Dispersal dynamics in food webs. American Naturalist, Dryad Data Repository, http://doi.org /10.5061/dryad.06bb5.

$\rightarrow$ Morlon, H., G. Chuyong, R. Condit, S. Hubbell, D. Kenfack, D. Thomas, R. Valencia, and J. L. Green. 2008. A general framework for the distance-decay of similarity in ecological communities. Ecology Letters 11:904-917.

$\rightarrow$ Mouquet, N., and M. Loreau. 2003. Community patterns in sourcesink metacommunities. American Naturalist 162:544-557.
Murdoch, W. W., C. J. Briggs, and R. M. Nisbet. 2003. Consumerresource dynamics. Princeton University Press, Princeton, NJ.

Nekola, J. C., and S. Peter. 1999. The distance decay of similarity in biogeography and ecology. Journal of Biogeography 26:867-878.

Pillai, P., A. Gonzalez, and M. Loreau. 2011. Metacommunity theory explains the emergence of food web complexity. Proceedings of the National Academy of Sciences of the USA 108:19293-19298.

Poisot, T., E. Canard, D. Mouillot, N. Mouquet, and D. Gravel. 2012. The dissimilarity of species interaction networks. Ecology Letters 15:1353-1361.

Polis, G. A. 1991. Complex trophic interactions in deserts: an empirical critique of food web theory. American Naturalist 138:123155.

Rezende, E. L., E. M. Albert, M. A. Fortuna, and J. Bascompte. 2009. Compartments in a marine food web associated with phylogeny, body mass, and habitat structure. Ecology Letters 12:779-788.

Shurin, J. 2001. Interactive effects of predation and dispersal on zooplankton communities. Ecology 82:3404-3416.

$\rightarrow$ Smith, D., J. K. Bailey, S. M. Shuster, and T. G. Whitham. 2011. A geographic mosaic of trophic interactions and selection: trees, aphids and birds. Journal of Evolutionary Biology 24:422-429.

Starý, P. 2006. Aphid parasitoids of the Czech Republic (Hymenoptera: Braconidae, Aphidiinae). Academia, Prague.

Thibaut, L., and S. Connolly. 2013. Understanding diversity-stability relationships: towards a unified model of portfolio effects. Ecology Letters 16:140-150.

Thuiller, W., T. Münkemüller, S. Lavergne, D. Mouillot, N. Mouquet, K. Schiffers, and D. Gravel. 2013. A road map for integrating ecoevolutionary processes into biodiversity models. Ecology Letters 16:94-105.

Vanpeteghem, D., and B. Haegeman. 2010. An analytical approach to spatio-temporal dynamics of neutral community models. Journal of Mathematical Biology 61:323-357.

Wang, S., and M. Loreau. 2014. Ecosystem stability in space: $\alpha, \beta$ and $\gamma$ variability. Ecology Letters 8:1175-1182.

Associate Editor: J. Nathaniel Holland Editor: Judith L. Bronstein 